\title{
Primary Paranasal Maxillary Hematoma: Clinical Characteristics, Diagnosis and Surgical Treatment
}

\author{
Natalia Franco de Oliveira e Oliveira', Murilo Martinez Matheus', Fernando Mosquera Jaramillo', \\ Mauricio José Bittar ${ }^{3}$, José Carlos Guilarde Pacheco ${ }^{4}$, Luis Claúdio Lopes Correia da Silva ${ }^{5}$, \\ André Luis do Valle De Zoppa ${ }^{5}$ \& Rodrigo Romero Corrêa ${ }^{5}$
}

\begin{abstract}
Background: The equine paranasal sinus have a complex anatomy and large compartiments. For this reason, deseases that affect these structures may develop for long periods before the animal show any clinical signs, making it difficult to stablish a definitive diagnosis and institute an adequate treatment. Usually, maxillary hematomas reports come from progression of ethmoidal hematomas, and the descriptions of primary maxillary hematomas are rare. This study aims to report the clinical features, diagnosis and treatment of a case of a maxillary hematoma not associated with ethmoidal turbinates. Case: An 8-year-old male horse, Mangalarga Paulista, was referred to the Centro de Apoio ao Ensino e Pesquisa FMVZUSP with history of nasal bleeding for over a year. The animal presented deformity on the right side of the face, with significant volume increase on the maxillary bone region and dull sound at percussion, in addition to great painfull sensibility when palpated. The right nare had no airflow, suggesting complete obstruction of the right nasal cavity. In order to better evaluate, endoscopic and radiographic exams were performed. At the radiographic exam, in ventrodorsal projection, it was observed an increase of volume and radiopacity, occupying the right antimer of the nasal cavity, with left nasal septum deviation. In the right dorsoventral oblique projection, it was observed the filling of the rostral and caudal maxillary sinus with the content radiopacity as previously described. At endoscopy exam of the right nasal cavity, it was observed a rounded greenish structure on the middle meatus The diagnosis of maxillary paranasal sinus cist was suggested and surgical removal, through maxillary sinusotomy was recommended. During the surgical procedure, it was noted that the structure previously observed, was not a cavitary organization filled with liquid, but a deorganized, dark and friable mass, like a hematoma. A fragment was sent to histopathological evaluation, which revealed the presence of intact eythrocytes interspersed by fibrilar eosinophilic material (fibrin). There were no indications of an infectious or neoplastic process. The diagnosis of maxillary hematoma was concluded. Forty days after admission, the patient had no surgical complications and showed adequate respiratory flow, at which point the animal was discharged.

Discussion: Primary maxillary hematomas are rare on equines. Although benign, it has destructives and expansives characteristics, and for this reason, it can be confused with malignant tumor formation. It has unknown ethiology, and the clinical signs observed are diverse and non specific, which makes it hard to stablish a definitive diagnosis based only on the clinical manifestations of the animal. In this particular case presented, the diagnosis was defined from the hitophatological examination. Initially the image exams (radiography and endoscopy) were usefull to stablish differential diagnosis, as well as the extention of the sinus mass. The surgery was performed with the animal on quadrupedal position, under sedation and local anethesia, in order to dimish haemorrhage occurance during the procedure. This paper describes a primary maxillary hematoma on a horse, characterizing it as to the clinical and pathological manifestations, as well as its surgical treatment. Although rare descriptions and with non specific clinical signs, it is suggested that its occurrence is superior to that described in the literature.
\end{abstract}

Keywords: equine, maxillary hematoma, obstruction, paranasal sinus, sinusotomy.

DOI: $10.22456 / 1679-9216.93266$ 


\section{INTRODUCTION}

The paranasal sinus of horses are subject to a wide variety of diseases, which although rare, represents a challenge to the veterinarian. The complex anatomy of these structures and their large size allow the diseases to develop for long periods (weeks to months) before the animal presents clinical signs, such as nasal discharge and increase of volume, which makes diagnosis and treatment of these injuries even more difficult [16]. The sinusal diseases can be classified as primary, when no apparent cause is defined to explain its development, or secondary to other injuries, such as apical infections on molar and premolar teeth, persistents oronasal fistula, sinusal cists, neoplasms, fungal infections, trauma and progressive ethmoidal hematoma [14,15]. Maxillary hematoma is usualy reported as a disease that progressed from an ethmoidal hematoma (progressive ethmoidal hematoma) leading to secondary sinusitis. Whereas the maxillary hematoma not associated with the ethmoidal turbinates corresponds to a rare disease on equines, with unknown ethiology [1]. The diagnosis usually is defined based on histopathological exams, and imaging techniques help to stablish mass extension. This paper aims to report a

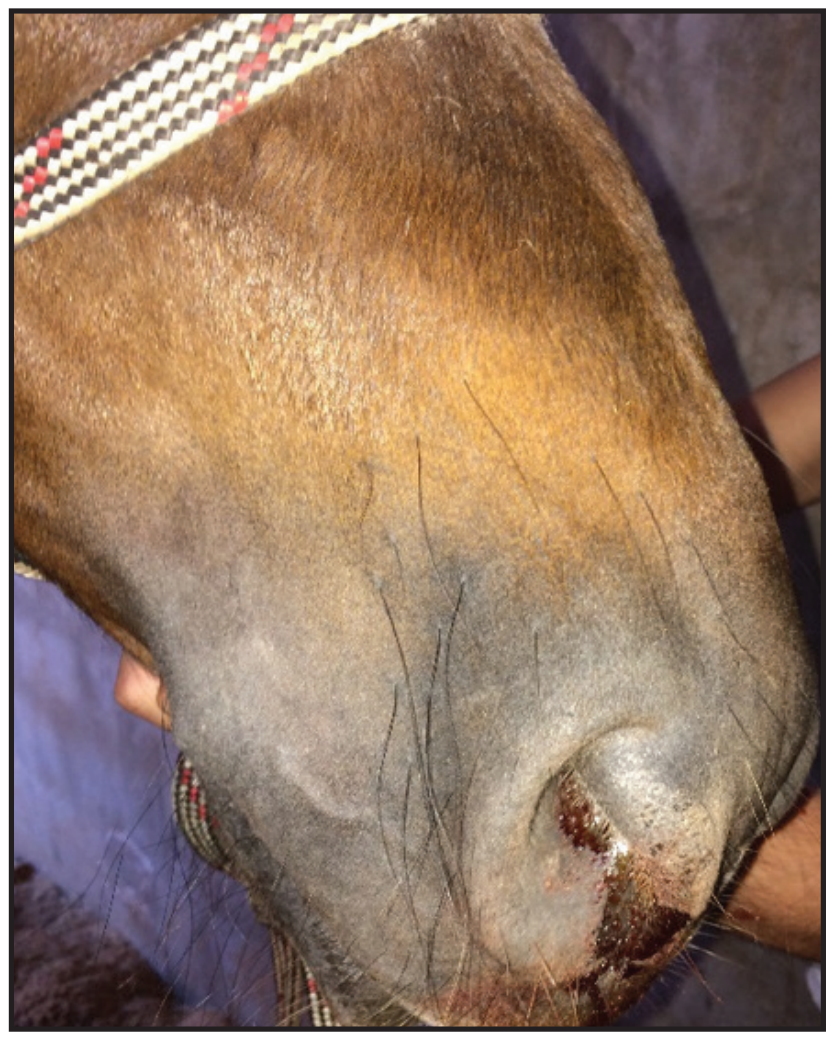

Figure 1. Image of the right nortril of the animal at admission. It is noted a small amount of bloody secretion on the right nostril case of a primary maxillary hematoma, characterizing it clinically, as well as its treatment.

\section{CASE}

An 8-year-old equine, male, Mangalarga Paulista, was reffered to the Centro de Apoio ao Ensino e Pesquisa (CAEP) FMVZ-USP. The horse owner reported that the animal had presented bleeding on its right nostril one year before, on the left nostril 6 months before and started with a respiratory noise 3 months before referral. The owner reported as well that the animal startet presenting an increase of volume on the right maxillar region one month before. At the field endoscopic and radiographic evaluation were performed, which suggested presence of a right maxillary paranasal cist.

At inicial physical evaluation the animal presented a cardiac frequency of $64 \mathrm{bpm}$, respiratory rate of $30 \mathrm{rmpm}$, temperature of $37.6^{\circ} \mathrm{C}$, capillary filling time of $2 \mathrm{~s}$, and mucosa normally colored. The animal presented respiratory noise and bloody secretion on the right nostril (Figure 1), right face deformity showing a significative increace of volume on the right maxillary paranasal sinus region, which presented points of fluctuation on its central and ventral region (Figure 2).

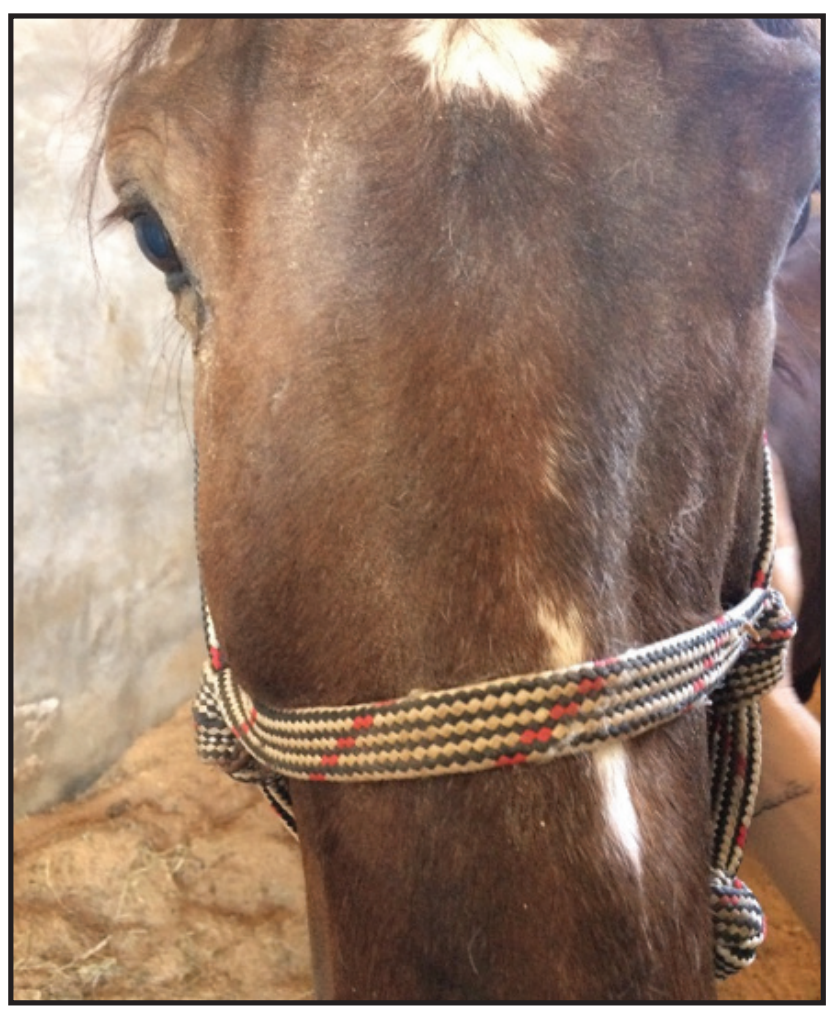

Figure 2. Frontal image of the head of the patient at admission. It is observed a large increase of volume on the right maxillary region, impeding the visualization of the facil crest. 


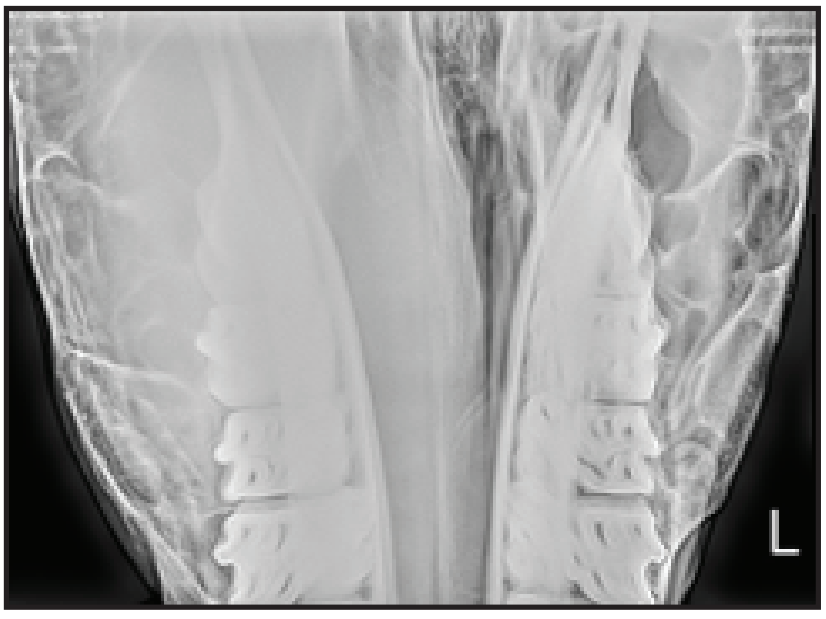

Figure 3. Radiographic image, ventrodorsal projection. It is observed increase of radipacity on right antimer, involving maxillary paranasal sinus and nasal septum deviation to the left

Presented dull sound at persussion and pain when manipulated. The right nostril had no airflow, suggesting complete obstruction by the mass.

To evaluate the animal, endoscopic and radiographic exams were performed. At the radiographic images, on the ventrodorsal projection, it was observed an increase of volume with filling of greater radiopacity, occupying the right antimer of the right nasal cavity, with nasal septum deviation to the left (Figure 3 ). On the right dorsoventral oblique projection it was observed filling of the rostral and caudal paranasal sinuses with a content as previously described (Figure 4). No apparent alteration on ethmoidal turbinates were observed on both sides. At rinoscopy it was noted an abnormal bulging of the nasal septum, being projected to the left side, with a luminal size reduction of the left antimer. When the right nasal cavity was evaluated, it was observed a greenish rounded structure (Figure 5), localized on the middle meatus, shifting the dorsal conchae dorsally, and blood throughout all endoscopy path. The ventral meatus was parttially obstructed allowing endoscope passage. After this exam, it was suggested the diagnosis of right maxillary paranasal cist, and surgical removal through sinusotomy was recommended.

Sinusotomy was performed 5 days after admission. Previously to the surgery the animal received flunixin meglumin $\left(\mathrm{EV}, 1.1 \mathrm{mg} / \mathrm{kg}-\text { Flumax }^{\circledR}\right)^{1}$ and enrofloxacin (IV diluted in $250 \mathrm{~mL}$ of physiologic solution, $5 \mathrm{mg} / \mathrm{kg}$ - Chemitril $\left.10 \%{ }^{\circledR}\right)^{2}$. The horse was sedated with detomidin $\left(10 \mu \mathrm{g} / \mathrm{kg} \text {, bolus - Detomidin }{ }^{\circledR}\right)^{3}$ and $0.1 \mathrm{mg} / \mathrm{kg}$ bolus of morfine $\left(\text { Dimorf }^{\circledR}\right)^{4}$. After first sedated, it was maintained in continuous infusion of

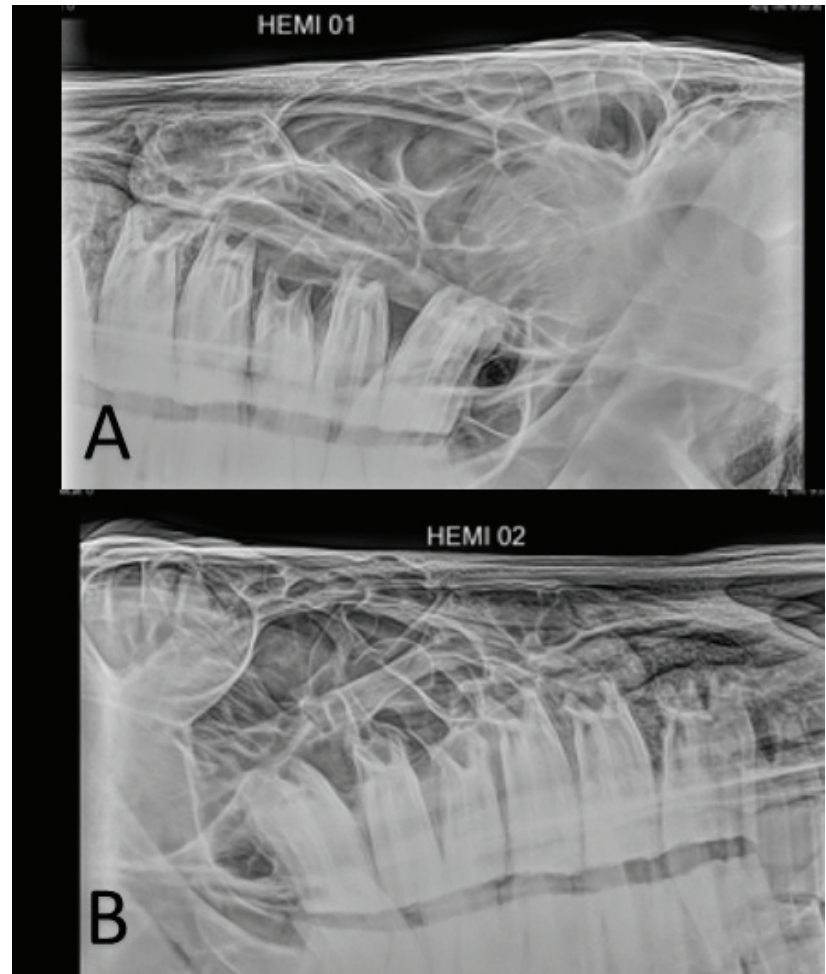

Figure 4. Radiographic image, right (A) and left (B) oblique dorsoventral projection. A- It is observed increase of radiopacity on rostral and caudal maxillary paranasal sinus. Contralateral comparative image in which can be observed normal radiopacity on the left paranasal sinus.

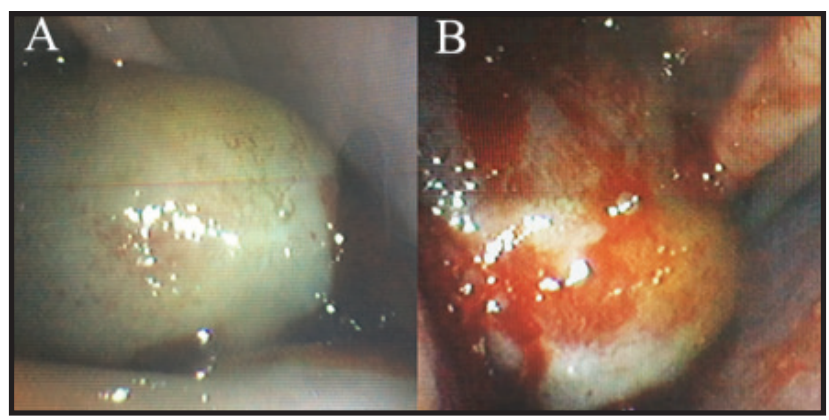

Figure 5. Endoscopic image of the right nasal cavity. A- It is observed a greenish rounded structure, located between the middle meatus and the dorsal conchae. B- The same structure visualized from a different angle, involved by blood.

detomidin at a rate of $10 \mu \mathrm{g} / \mathrm{kg} / \mathrm{h}$. The animal was submitted to regional anesthesia (right maxillar nerve) with $10 \mathrm{~mL}$ of lidocaine $2 \%$ (cloridrato de Lidocaína $\left.{ }^{\circledR}\right)^{5}$ and $10 \mathrm{~mL}$ of bupivacaine (cloridrato de bupivacaína $\left.{ }^{\circledR}\right)^{5}$, and the skin was blocked at incision line using $10 \mathrm{~mL}$ of lidocaine.

After preparing surgically the affected site, the sinusotomy was performed. The bone flap was made based on the technique described by Nickels [6]. In the described case, the mass had compromised the bone structure, turning it into a thin bone layer, making the osteotomy easy to perform. Due to bone compromise, 
the surgeon chose to remove the bone flap, leaving only skin and periosteum to reconstitute.

After folding the flap dorsally, the mass was visualized and consisted on a big structure invading the rostral and caudal paranasal sinuses, the ventral conchae sinus and the dorsal conchae sinus. It was observed a shining greenish capsule, aparentlly of fibrous tissue, surrounding the mass. In its interior there were a brownish structure with clot consistence. There were no signs of local infection.

Removal of the mass as a unity was not possible due to the difficult acces of the paranasal sinuses. Thus, the mass was fragmented and removed by pieces. It was observed destruction of the paranasal septs, thus the sept between rostral and caudal paranasal sinuses was not observed, as well as the ventral conchal bulla. It was not observed any alteration of the ethmoidal turbinates whatsoever. After mass removal, it was performed

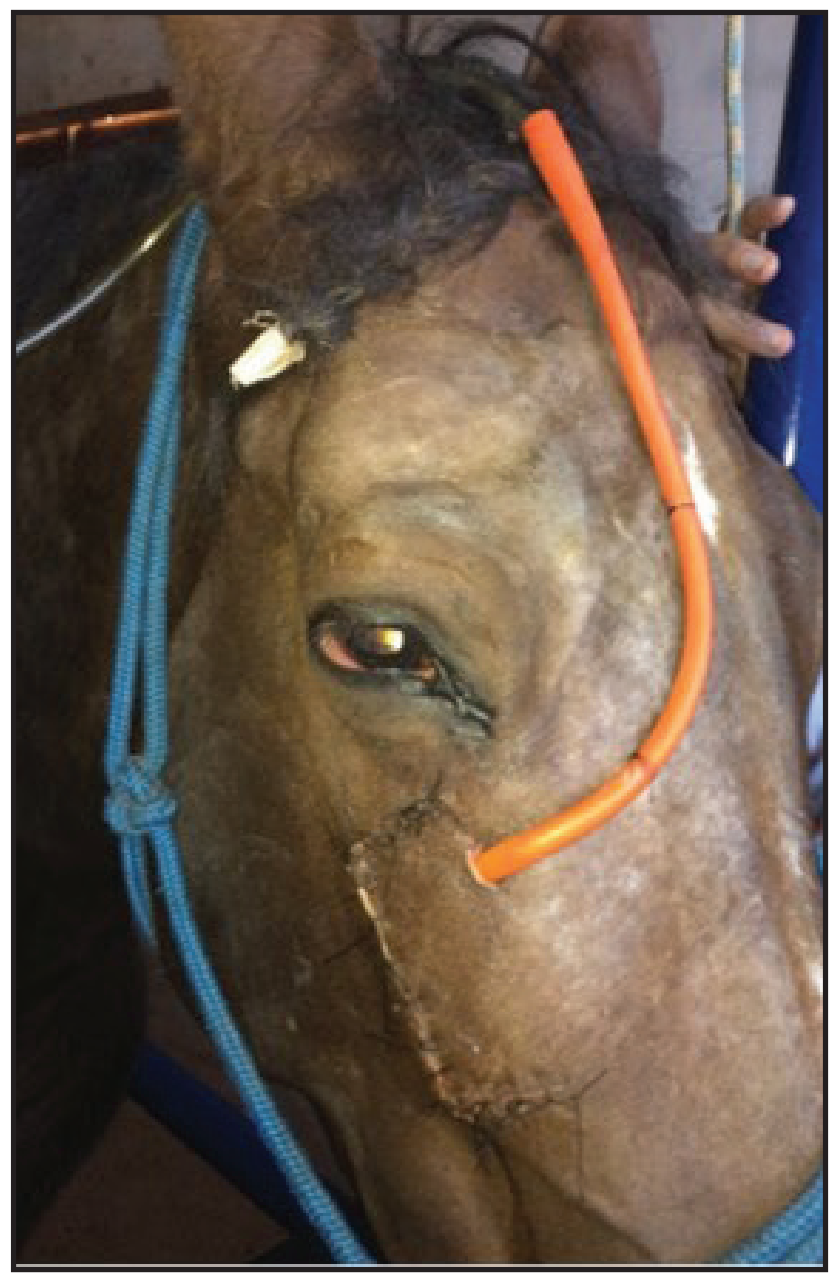

Figure 6. Right maxillary region on the third post-operative day. It can be seen the sinusotomy region with volume increase, as well as the Pezzer catheter introduced into the caudal maxillary sinus near the caudal and dorsal limits of the surgical access. a local curettage and a new acess with aproximatelly 1 $\mathrm{cm}$ of diameter was made, over the caudal maxillary sinus. This new access had the purpose to allocate a Pezzer catheter $n^{\circ} 24$ (Silkolatex $\left.^{\circledR}\right)^{6}$. An amount of gauze, impregnated with topic PVP-I $\left(\text { Riodeine }^{\circledR}\right)^{7}$, was left under pressure at this access in order to bleeding restrain, and its extremity was left outside of the site to future remove it. Periosteum and subcutaneous was sutured with continuous simple pattern and skin was sutured with interrupted simple pattern. Two days after surgery, the gauze was removed and the Pezzer catheter ${ }^{6}$ was inserted in order to begin sinus washes.

A fragment of the mass was collected for histopathological analyses, which revealed the presence of intact and degenerate erythrocytes interspersed by fibrillar eosinophilic material (fibrin), discret amount of polymorphonuclear leukocytes and mainly macrophages with gold-granular to brownish intracytoplasmatic pigment (hemosiderin) and large amount of free gold-yellow pigment. There were no indications of infectious or neoplastic process. The diagnosis of maxillary hematoma was concluded

The post operative treatment consisted on enrofloxacin $(5 \mathrm{mg} / \mathrm{kg} \mathrm{IV}$, diluted on $250 \mathrm{~mL}$ of physiologic solution) for 15 days, flunixin meglumine (IV, $1.1 \mathrm{mg} / \mathrm{kg}$ ) for 3 days, as well as right maxillary sinus washes (Figure 6) with $0.1 \%$ PVP-I solution initially 4 times a day (first 18 days) followed by 2 times a day (last 15 days).

The patient was discharged 40 days after admission, no longer presenting any clinical signs of sinusal disease. The owner was contacted 9 months after discharge, and the animal had no clinical signs and had completelly returned to its physical activities.

\section{DISCUSSION}

There are few reports describing progressive maxillary hematoma in equines [1]. Usually, descriptions refer to ethmoidal hematomas, with a reported prevalence of this disease of 3.5-8.2\% among cases of sinonasal diseases $[3,14]$.

When there is involvement of paranasal sinuses, it is usually due to ethmoidal hematoma progression, as reported by Quinn et al. [10] who described the appearance of progressive ethmoidal hematoma located in the rostral maxillary sinus in 2 of the 60 cases of animals submitted to frontonasal flap surgery for the treatment of sinonasal diseases. 
There is a report of small hematomas on the paranasal sinuses distant from the ethmoidal turbinates. However, because the histopathological examination was not performed, the diagnosis could not be confirmed [2]. In other reports, the authors described the successful removal of a progressive hematoma affecting the rostral and caudal maxillary sinuses [12] and three cases of bilateral sinonasal hematoma in horses [1].

In humans, progressive maxillary hematoma is also reported as rare disease. It has a benign character, although its local destructive behavior, and, for this reason, it can be confused with a malignant tumor formation $[13,17]$. The hematoma mass has an expansive feature and compresses adjacent structures, leading to bone erosion. It is believed that its progressive nature occurs due to the presence of the fibrous capsule surrounding the blood clot, which prevents reabsorption of the hematoma. Future bleeds result in increased pressure within the organized hematoma, leading to further expansion and compression of adjacent structures $[13,17]$.

Considering the similarity with tumor masses, some authors have reported the possibility that some possible diagnostic errors have been committed, and thus, the disease underdiagnosed [11,17], which also raises this possibility for equine medicine. In addition, due to the rarity of the disease in human medicine, there is still no consensus in its nomenclature. The most commonly used names are organized hematoma and mass similar to hematoma, in addition to tumors such as hemangiosarcoma, which may be confused or considered $[11,17]$. For differentiation between hematoma and neoplasia, histopathological examination becomes indispensable and reduces diagnostic errors $[11,13]$.

The etiology of organized maxillary hematoma is still unknown in both humans and horses. In humans the negative spiral theory was suggested [9]. Initially a blood clot accumulates in the paranasal sinus space, from a bleeding for any reason, including hemangioma formation, facial trauma or inflammation. Subsequently, necrosis, fibrosis and hyalinization occur, and neovascularization is developed as part of the biological healing process. As result, the blood flow in the newly formed vessels becomes slow, providing dilatation of these structures, which leads to new bleeding, perpertuating the cycle. This theory seems to be well accepted in human medicine $[7,8]$.
In equine medicine there is also no formulated etiology. Barker and colleagues [1] cited a hypothesis regarding the etiology of the disease, which is believed to originate from recurrent hemorrhages of the submucosa associated with the ethmoidal turbinates. They also suggested that the animals evaluated in their work could present generalized inflammation in the upper respiratory tract, and possibly systemic disorders, such as coagulopathies or genetic vascular fragility.

The clinical signs associated with maxillary hematoma are diverse. The horse in question initially presented unilateral epistaxis, progressing to deformity in the maxillary region, with pain when manipulated the region and total obstruction of the right nasal cavity, confirming the expansive and destructive character of the mass. The nasal bleeding did not stoped until the mass was removed. These clinical signs corroborate with several other reports in human medicine. Epistaxis was the most frequent clinical sign in the articles surveyed $[5,7,8,11,18]$. The authors also cited nasal obstruction and increased local volume as being commonly observed signs.

In a retrospective study which evaluated the findings of 200 cases of paranasal sinus disease in horses, when the animals that had paranasal cysts, neoplasia and ethmoidal hematoma (all differential diagnosis of maxillary hematoma) were analyzed, one of the most observed signs was nasal discharge, corresponding to $81 \%, 50 \%$ and $100 \%$, respectively. Forty-three percent of the animals with ethmoidal hematoma presented epistaxis, and nasal obstruction was confirmed in $31 \%$ of the animals with cyst and $40 \%$ of the animals with neoplasia. This article shows the inespecificity of the clinical signs presented in diseases of the paranasal sinuses, bringing the importance of the establishment of differential diagnosis, complementary imaging studies and histopathological examination [3]. In a report of bilateral maxillary hematoma, the three animals referred to the center presented serosanguinolent to mucopurulent nasal discharge and intermittent epistaxis. They also had some degree of respiratory distress [1].

The diagnosis of the reported case was defined based on histopathological examination. Initially the imaging exams (radiography and endoscopy) served to establish differential diagnosis, as well as the mass extension. As previously mentioned, the possibility of errors in diagnosis is great considering not only the 
rarity of maxillary hematoma, but also the possibility of other diseases that present similar clinical signs, such as maxillary cysts and tumors. For this reason, the diagnosis should not be based only on the signs presented, but also on imaging and histological exams.

The difficulty found in establishing a diagnosis based only on the image occurs due to its appearance that can range from a benign process, such as mucocele, paranasal cyst, to a malignant process, such as aggressive neoplasias. However, the importance of radiographic examination is based on the determination of mass extension and invasion of anatomical areas [5]. Although it is important, the radiographic examination has little efficacy when it comes to masses of smaller sizes, due to the overlap of anatomical structures [14]. For this reason, when available, computed tomography is the imaging technique of choice for diagnostic assistance [1].

In this case, the endoscopic examination served as an aid to evaluate the extent of the mass and its external characteristics. It had a well-defined capsule of greenish color, similar to that described in cases of ethmoidal hematoma [4].

Histopathological examination has great value in the diagnosis. There are three histological features that correspond to organized hematoma: dilated vessels, hemorrhage and presence of fibrin. These three characteristics are usually found in separate sessions within the same mass. For this reason, multiple areas should be analyzed for diagnosis [17]. For the complete discard of malignancy, neoplastic cells can not be evidenced $[5,8,17]$. The histopathological findings of the fragments submitted for analysis are consistent with the above cited articles concerning humans.

Mass excision was performed with the animal in standing position in order to reduce the risks of general anesthesia, as well as to reduce local hemorrhage. The removal of the bone flap of the maxillary sinus was necessary for better visibility of the mass and affected paranasal sinuses, since the mass involved several paranasal compartments of the equine in question.

The location of the mass and the anatomy of paranasal sinuses hindered the complete formation withdrawal. For this reason, the formation was removed by fragments and not in block. In addition, residual portions of the mass were left in the rostral regions of the ventral and dorsal conchal sinuses. Barker et al. [1] reported the same difficulty in complete mass withdrawal. According to them, the fact that the formation can't be completely excised facilitates the relapse of the disease, which can vary from 14-44\% of cases. Until the moment of the present report, the animal did not show recurrence and was able to return to its physical activities.

This paper reports the occurrence of a progressive maxillary hematoma not associated with the ethmoidal turbinates, bringing attention to the inclusion of this disease in the diferential diagnosis of paranasal sinus diseases. Its expansive and destructive character resembles a malignant tumor, and histopathological examination is necessary for the accurate establishment of the diagnosis. Its surgical excision was successfully performed by sinusotomy, and the postoperative evolution was adequate to the expectations of the veterinary team and the owner.

\section{MANUFACTURERS}

1JA Saúde animal. Patrocínio Paulista, SP, Brazil. ${ }^{2}$ Chemitec Agro-Veterinária Ltda. São Paulo, SP, Brazil. ${ }^{3}$ Syntec. Santana de Parnaíba, SP, Brazil ${ }^{4}$ Laboratório Cristália. Itapira, SP, Brazil.

${ }^{5}$ Hipolabor Farmacêutica Ltda. Belo Horizonte, MG, Brazil.

${ }^{6}$ Teleflex Medical. Kamubting, Malasia.

${ }^{7}$ Indústria Farmacêutica Rioquímica Ltda. São José do Rio Preto, SP, Brazil.

Declaration of interest. The authors report no conflicts of interest. The authors alone are responsible for the content and writing of the paper.

\section{REFERENCES}

1 Barker W.H.J., Perkins J.D. \& Witte T.H. 2013. Three horses with bilateral sinonasal progressive haematomas not associated with the ethmoidal labyrinth. Equine veterinary Education. 25(10): 503-507.

2 Cook W.R. \& Littlewort M.C. 1974. Progressive haematoma of the ethmoid region in the horse. Equine Veterinary Journal. 6: 101-108.

3 Dixon P.M., Parkin T.D., Collins N., Hawkes C., Townsend N., Tremaine W.H., Fisher G., Ealey R. \& Barakzai S.Z. 2011. Equine paranasal sinus disease - a long-term study of 200 cases (1997-2009): ancillary diagnostic findings and involvement of the various sinus compartments. Equine Veterinary Journal. 44: 267-271. 
4 Greet T.R. 1992. Outcome of treatment in 23 horses with progressive ethmoidal haematoma. Equine Veterinary Journal. 24: 468-471.

5 Lim M., Lew-Gor S., Beale T., Ramsay A. \& Lund V.J. 2008. Maxillary sinus haematoma. The Journal of Laryngology \& Otology. 122: 210-212.

6 Nickels F.A. 2006. Nasal Passages and Paranasal Sinuses. In: Auer J.A. \& Stick J.A. (Eds). Equine Surgery. 3rd edn. St. Louis: Elsevier Saunders, pp.533-544.

7 Ohta N., Watanabe T., Ito T., Kubota T., Suzuki Y, Ishida A., Aoyagi M., Matsubara A., Izuhara K. \& Kakehata S. 2013. Clinical and Pathological Characteristics of Organized Hematoma. International Journal of Otolaryngology. Article ID 539642, pp.1-6.

8 Omura G., Watanabe K., Fujishiro Y., Ebihara Y., Nakao K. \& Asakage T. 2010. Organized hematoma in the paranasal sinus and nasal cavity - Imaging diagnosis and pathological findings. Auris Nasus Larynx. 37: 173-177

9 Ozaki M., Sakai S. \& Ikeda H. 1977. Hemangioma of the nasal cavity and sinuses - a report of twenty five cases. Otolaryngology Head Neck Surgery. 49: 53-58.

10 Quinn G.C., Kidd J.A. \& Lane J.G. 2005. Modified frontonasal sinus flap surgery in standing horses: surgical findings and outcomes of 60 cases. Equine Veterinary Journal. 37: 138-142.

11 Song H.M., Jang Y.J., Chung Y.S. \& Lee B.J. 2007. Organizing hematoma of the maxillary sinus OtolaryngologyHead and Neck Surgery. 136: 616-620.

12 Sullivan M., Burrell M.H. \& McCandlish I.A. 1984. Progressive haematoma of the maxillary sinus in a horse. Veterinary Record. 114: 191-192.

13 Tabaee A., Kacker A. 2002. Hematoma of the maxillary sinus presenting as a mass - a case report and review of literature International Journal of Pediatric Otorhinolaryngology. 65: 153-157.

14 Tremaine W.H. \& Dixon P.M. 2001. A long-term study of 277 cases of equine sinonasal disease Part I: details of horses, historical, clinical and ancillary diagnostic findings. Equine Veterinary Journal. 33: 274-282.

15 Tremaine, W.H. \& Freeman D.E. 2007. Disorders of the paranasal sinuses. In: McGorum B.C., Robinson N.E., Dixon P.M. \& Schumacher J. (Eds). Equine Respiratory Medicine and Surgery. Oxford: Elsevier Saunders, pp.393-408.

16 Waguespack R.W. \& Taintor J. 2011. Paranasal sinus disease in horses. Compendium: continuing education for veterinarians. 33(2): 1-12.

17 Yagisawa M., Ishitoya J. \& Tsukuda M. 2006. Hematoma-like mass of the maxillary sinus. Acta Oto-Laryngologica. 126: $277-281$.

18 Yokoi H., Arakawa A., Matsumoto F., Yokoi N., Ikeda K. \& Kohno N. 2014. Organized hematoma of the maxillary sinus: A clinicopathologic study of 5 Cases. Ear, Nose \& Throat Journal. 93(2): 23-26. 